\title{
Chime's Laws for Distribution of Planets, Moons and Planetary Ring-Systems: Critical Examination of Their Predictions and the Implications
}

\author{
Chime Peter Ekpunobi ${ }^{1,2,3}$ \\ ${ }^{1}$ Department of Theoretical Astronomy, Charles-Monica Observatory, Enugu, Nigeria \\ ${ }^{2}$ Department of Internal Medicine, Enugu State University of Science and Technology, Enugu, Nigeria \\ ${ }^{3}$ Department of Medicine, ESUT Teaching Hospital, Enugu, Nigeria
}

\section{Email address:}

pe4chyme@yahoo.com

\section{To cite this article:}

Chime Peter Ekpunobi. Chime's Laws for Distribution of Planets, Moons and Planetary Ring-Systems: Critical Examination of Their Predictions and the Implications. American Journal of Astronomy and Astrophysics. Vol. 6, No. 3, 2018, pp. 57-71. doi: 10.11648/j.ajaa.20180603.12

Received: August 5, 2018; Accepted: August 22, 2018; Published: October 15, 2018

\begin{abstract}
Study Aim: The problem as to whether planets, moons and planetary ring-systems are randomly or non-randomly distributed in the solar system has not been resolved. In this article, the predictions of Chime's laws for distribution of bodies were critically examined in order to see if planets, moons and planetary ring-systems were randomly or non-randomly distributed in the solar system. Method: Equations formulated based on the distribution of the known planets, moons and planetary ring-systems in the solar system, were used to predict the distribution of these bodies in all the planetary orbits of the solar system. The predictions made by those equations were then compared with observations. Result: The solar system is a 14-orbit system which has between 9 and 13 formed planets, between 190 and 248 formed moons and 6 planetary ringsystems. Many Jupiter's moons are missing. About three Uranus' moons await discovery. Pluto is Planet 10 which was predicted to have 6 moons and a planetary ring-system. Planet Eleven, predicted to have 2 moons and a planetary ring-system, has not yet been discovered. The non-random distribution of planets, moons and planetary ring-systems in the solar system favoured a formation process that was not prone to chance. There are serious flaws in the IAU definition of planet, which was why it was unable to recognize that Pluto is a Planet. Conclusion: This study has shown that planets, moons and planetary ringsystems are non-randomly distributed in the solar system. The simplicity, elegance and Fibonacci-friendliness of the beta total orbital bodies distribution laws make them very attractive. No standard ring-system or any more moons are expected to be discovered in Section 2.1.1 of the solar system. Astronomers should, therefore, channel their energies and limited resources towards Section 2.1.2 of the solar system where it is necessary to resolve such puzzles as the number of Jupiter's moons that are actually missing, the group of laws that is operative in the solar system and the fate of Warsawlene, the sixth moon of Pluto. There are serious flaws in the 2006 IAU definition of planet. Systematic theoretical explorations of Division 2.2 should commence with Orbit Eleven and its planet. Recommendations: Search for the three undiscovered moons of Uranus labeled Ezechi, Akanene and Ikechukwu in the order in which they will be discovered; search for the sixth moon of Pluto, Warsawlene; recognizing the planetary status of Pluto; serious efforts to discover Planet Eleven; and coming up with a new and better definition of planet.
\end{abstract}

Keywords: Chime's Laws, Total Orbital Bodies, Planet-Moon Bodies, Distribution of Planets, Distribution of Moons, Distribution of Ring-Systems

\section{Introduction}

One very important problem in astronomy that has not been resolved is whether planets, moons and planetary ring- systems are randomly or non-randomly distributed in the solar system. Mars' capture of asteroids to become moons (Phobos and Deimos), the Moon originating from collision between the Earth and Theia, and Pluto escaping from 
Neptune to become a planet are all random events. The hypotheses supporting these and other similar things in the literature implied that chance played some role in the formation of some of the moons and some of the planets of the solar system. The hypotheses also implied that planets, moons and planetary ring-systems are randomly distributed in the solar system. However, Chime's Laws for Distribution of Planets, Moons and Planetary Ring-Systems predicted that planets, moons and planetary ring-systems are non-randomly distributed in the solar system [1]. The objective of this article is to critically examine the predictions of Chime's Laws for Distribution of Planets, Moons and Planetary RingSystems and analyze their implications.

The method of formation of planets and moons in the solar system should impact on the nature of their distribution in the solar system, yet there has not been a consensus regarding the method of formation of some of the planets and some of the moons in the solar system. The speculated origin of Phobos and Deimos ranged from asteroids capture [2] to results of impact between Mars and another body [3]. In the case of the Moon, several hypotheses have been put forward to explain its origin. The most popular of these appears to be the impact hypothesis [4] which has been modified over the years. As regards the origin of Pluto, it was Lyttleton who first hypothesized in 1936 that Pluto was an escaped moon of Neptune [5]. What all these hypotheses have in common is that they connote chance occurrences regarding the origin of these bodies. And chance occurrences would obviously lead to random distribution of these bodies in the solar system, if those were indeed what happened. Although, there have been a lot of studies trying to understand the exact origin of these bodies, direct studies to determine whether planets, moons and planetary ring-systems are randomly or non-randomly distributed in the solar system appear to be very limited. That was why this author thought it necessary to undertake this study.

\section{Chime's Laws for Distribution of Planets, Moons and Planetary Ring-Systems}

\subsection{Chime's Laws for Distribution of Bodies}

Chime's laws for distribution of planets, moons and planetary ring-systems are simply known as Chime's laws for distribution of bodies or more simply as Chime's bodies laws [1]. The two basic Chime's laws for distribution of bodies are the alpha planet-moon bodies distribution laws which determine or describe the distribution of planets and moons in the solar system, and the beta total orbital bodies distribution laws which determine or describe the distribution of planets, moons and planetary ring-systems in the solar system [1]. The alpha total orbital bodies distribution laws are extension of the alpha planet-moon bodies distribution laws [1].

\subsection{The Proposed Regions, Divisions and Sections in the Solar System}

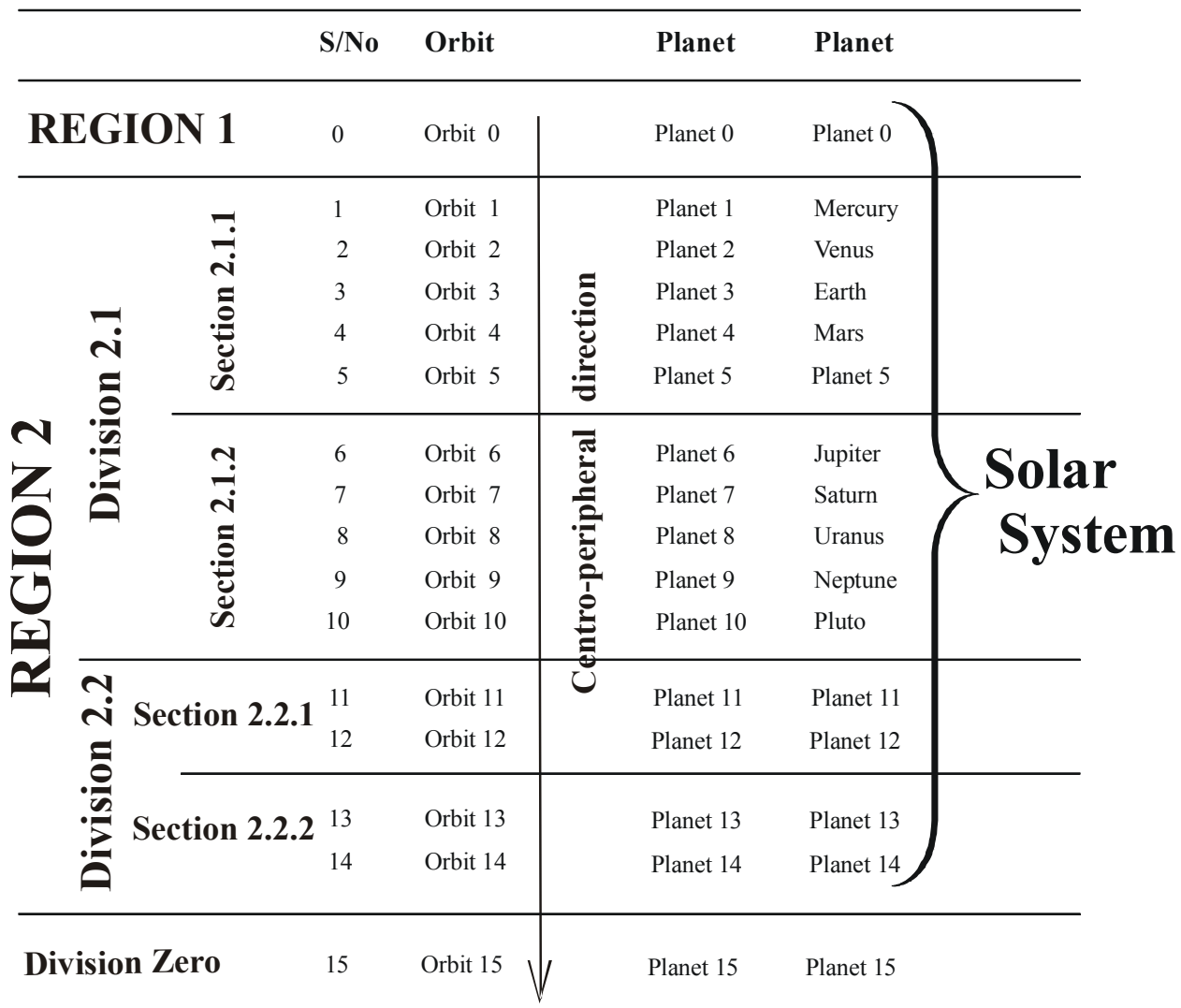


To be able to understand the laws for distribution of bodies, one needs to understand the regions, divisions and sections proposed for the solar system $[1,6,7]$, as well as the designations of planetary orbits and planets also proposed for the solar system [7, 8] which were used in the laws for distribution of bodies. These are illustrated schematically in Figure 1 and Figure 2. In the unidirectional, centro-peripheral designation of planetary orbits, the planetary orbits were numbered serially from Orbit Zero located at the center of the solar system to Orbit 15 which is believed to be located outside the solar system [7]. In the bidirectional designation of planetary orbits, Orbit Zero to Orbit Five were designated in the centro-peripheral direction, while the remaining planetary orbits were designated in the periphero-central direction [8]. In this second group, the former Orbit 15 became Orbit Zero, and the other planetary orbits were designated serially from the periphery towards the center as shown in the diagram (Figure 2). A planet was designated according to the planetary orbit in which it was located whatever designation was used $[7,8]$.

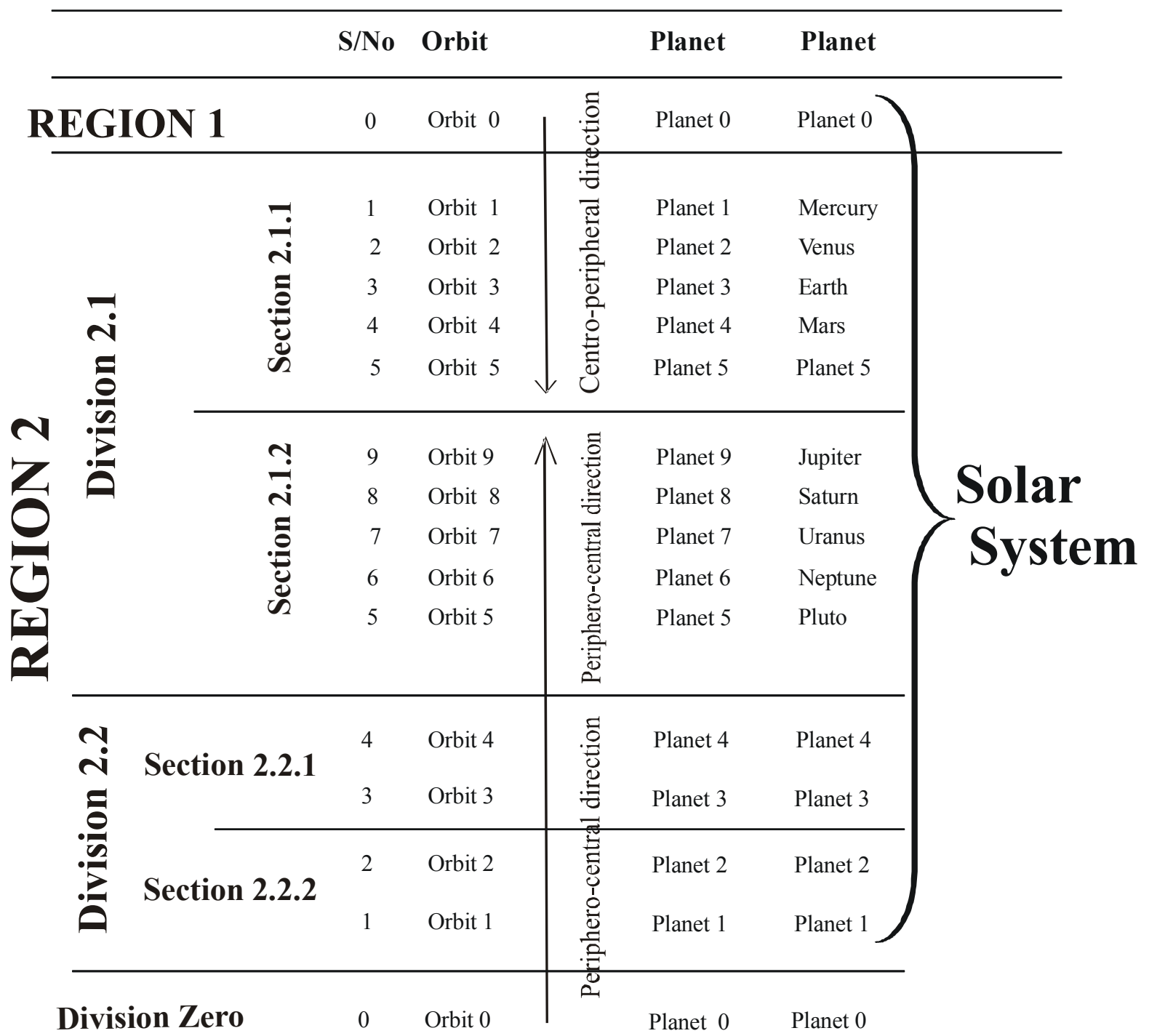

Figure 2. Bidirectional designation of planetary orbits and planets (centro-peripheral designation and periphero-central designation). Adapted with permission [8].

Using the unidirectional centro-peripheral designation, the sectional distribution of planetary orbits is as provided in Figure 3. 


\begin{tabular}{|l|l|l|l|}
\hline \multicolumn{2}{|l|}{ Solar system } & Section & Orbit \\
\hline Region & Division & & Orbit Zero \\
\hline Region 1 & & & \\
\hline & & Section 2.1.1 & Orbit 1, Orbit 2, Orbit 3, Orbit 4, Orbit 5 \\
\hline & & & \\
\hline & Division 2.1 & Section 2.1.2 & Orbit 6, Orbit 7, Orbit 8, Orbit 9, Orbit 10 \\
\hline Region 2 & & & \\
\hline & & Section 2.2.1 & Orbit 11, Orbit 12 \\
\hline & & & \\
\hline & Division 2.2 & Section 2.2.2 & Orbit 13, Orbit 14 \\
\hline & & & \\
\hline Region Zero & Division Zero & & Orbit 15 \\
\hline
\end{tabular}

Figure 3. The sectional distribution of planetary orbits in the solar system Adapted with permission [9].

\subsection{The Working Definitions and Computation of Planet-Moon Bodies and Total Orbital Bodies}

\subsubsection{The Working Definitions}

Formulation of Chime's laws for distribution of bodies was based on the currently available data on the solar system, i.e., the current distribution of planet, moons and planetary ringsystems in the solar system. First, the alpha planet-moon bodies distribution laws were developed on the bases of architectural models for the solar system [10]. This helped to find out which of the known planetary orbits of the solar system had all their moons discovered. The result showed that, with the exception of Obit 5 (the main asteroids orbit), the orbits of Section 2.1.1 of the solar system had all their moons discovered, i.e., Orbit 1(Mercury's orbit), Orbit 2(Venus' orbit), Orbit 3(Earth's orbit) and Orbit 4(Mars' orbit). Also, of all the orbits of Section 2.1.2 of the solar system, only Orbit 7(Saturn's orbit) and Orbit 9(Neptune's orbit) had all their moons discovered. Formulating the first beta total orbital bodies distribution laws was easy. But for the second beta total orbital bodies distribution laws, only the data from Orbit 7 and Orbit 9 were used because they were the only Section 2.1.2 planetary orbits with complete data.

The definitions of planet, moon, planetary ring-system, planet-moon bodies and total orbital bodies adopted for those analyses were as proposed in earlier publications [11, 12]. These are stated below.

Planet: "A planet is the principal body of its planetary orbit" $[11,12]$. (The body must be in a planetary orbit as well as being the principal body of that orbit).

Moon: "A moon is a satellite of a planet which exists as a compact body" [11]. "By this definition, the satellite of an asteroid is not considered to be a moon. It is just a satellite."

Planetary ring-system: "A planetary ring-system is a satellite of a planet which exists as a ring of gases, particles or rocky materials, or a combination of any of these, around the planet" [11]. Or "A planetary ring-system is a satellite of a planet which exists as a ring of non-compact body around the planet" [11]. "The ring-system of a planet is considered to be one body irrespective of its mass, size or number of its ringlets" [11].

Asteroid: "An asteroid is a major compact body in a primary star-centric orbit which is not a planet" [11].

Dwarf planet: The term dwarf planet as used in the 2006 definition of planet was considered a misnomer as being a planet is an all or none affair [11].

Planet-moon bodies: "Planet-moon bodies is the total number of planet and moons in a given planetary orbit" [11].

Total orbital bodies: "Total orbital bodies is the total number of planet, moons and planetary ring-system in a given planetary orbit" [11].

\subsubsection{Computation of Planet-Moon Bodies and Total Orbital Bodies}

Based on the working definitions, the known planet-moon bodies and total orbital bodies of the solar system were computed (Table 1).

Table 1. The planet-moon bodies and total orbital bodies of the first ten planetary orbits Adapted and modified with permission [13].

\begin{tabular}{|c|c|c|c|c|c|c|}
\hline S/No & Planetary orbit & No. of Planet & No. of Moons & No. of Planetary ring-system & Planet-moon bodies & Total orbital bodies \\
\hline 1 & $\begin{array}{l}\text { Orbit } 1 \\
\text { (Mercury's orbit) }\end{array}$ & 1 & 0 & 0 & 1 & 1 \\
\hline 2 & $\begin{array}{l}\text { Orbit } 2 \\
\text { (Venus' orbit) }\end{array}$ & 1 & 0 & 0 & 1 & 1 \\
\hline 3 & $\begin{array}{l}\text { Orbit } 3 \\
\text { (Earth's orbit) }\end{array}$ & 1 & 1 & 0 & 2 & 2 \\
\hline 4 & Orbit 4 & 1 & 2 & 0 & 3 & 3 \\
\hline
\end{tabular}




\begin{tabular}{|c|c|c|c|c|c|c|}
\hline S/No & Planetary orbit & No. of Planet & No. of Moons & No. of Planetary ring-system & Planet-moon bodies & Total orbital bodies \\
\hline 5 & $\begin{array}{l}\text { (Mars' orbit) } \\
\text { Orbit } 5 \\
\text { (Main asteroids' orbit) }\end{array}$ & 0 & 0 & 0 & 0 & 0 \\
\hline 6 & $\begin{array}{l}\text { Orbit } 6 \\
\text { (Jupiter's orbit) }\end{array}$ & 1 & 79 & 1 & 80 & 81 \\
\hline 7 & $\begin{array}{l}\text { Orbit } 7 \\
\text { (Saturn's orbit) }\end{array}$ & 1 & 62 & 1 & 63 & 64 \\
\hline 8 & $\begin{array}{l}\text { Orbit } 8 \\
\text { (Uranus' orbit) }\end{array}$ & 1 & 27 & 1 & 28 & 29 \\
\hline 9 & $\begin{array}{l}\text { Orbit } 9 \\
\text { (Neptune's orbit) }\end{array}$ & 1 & 14 & 1 & 15 & 16 \\
\hline 10 & $\begin{array}{l}\text { Orbit } 10 \\
\text { (Pluto's orbit) }\end{array}$ & 1 & 5 & 0 & 6 & 6 \\
\hline
\end{tabular}

\subsection{The Beta Total Orbital Bodies Distribution Laws}

\subsubsection{The First Beta total Orbital Bodies Distribution} laws:- (Law I)

Law IA: In Region One and Section 2.1.1 planetary orbits, the distribution of total orbital bodies is in the centroperipheral direction.

Law IB: In Region One and Section 2.1.1 planetary orbits, the total number of bodies in the $n t h$ planetary orbit is equal to the $n$th Fibonacci number where $\mathrm{n}$ is the serial number of the planetary orbit in the centro-peripheral direction.

$$
\mathrm{B}_{\mathrm{T}}=\mathrm{B}_{\mathrm{P}}+\mathrm{B}_{\mathrm{Mn}}+\mathrm{B}_{\mathrm{R}}=\mathrm{F}_{\mathrm{n}}
$$

$$
\text { Or } \quad \mathrm{B}_{\mathrm{T}}=\mathrm{F}_{\mathrm{n}}
$$

where $\mathrm{B}_{\mathrm{T}}$ is total orbital bodies

$B_{P}$ is number of planet which is usually one

$\mathrm{B}_{\mathrm{Mn}}$ is number of moons which may be zero or

a positive integer

$B_{R}$ is number of ring-system which may be zero or one $\mathrm{F}_{\mathrm{n}}$ is the nth Fibonacci number

$\mathrm{n}$ is the serial number of the planetary orbit in the centroperipheral direction

See Figure 4 for the predictions of the first beta total orbital bodies distribution laws for Orbit Zero to Orbit 5.

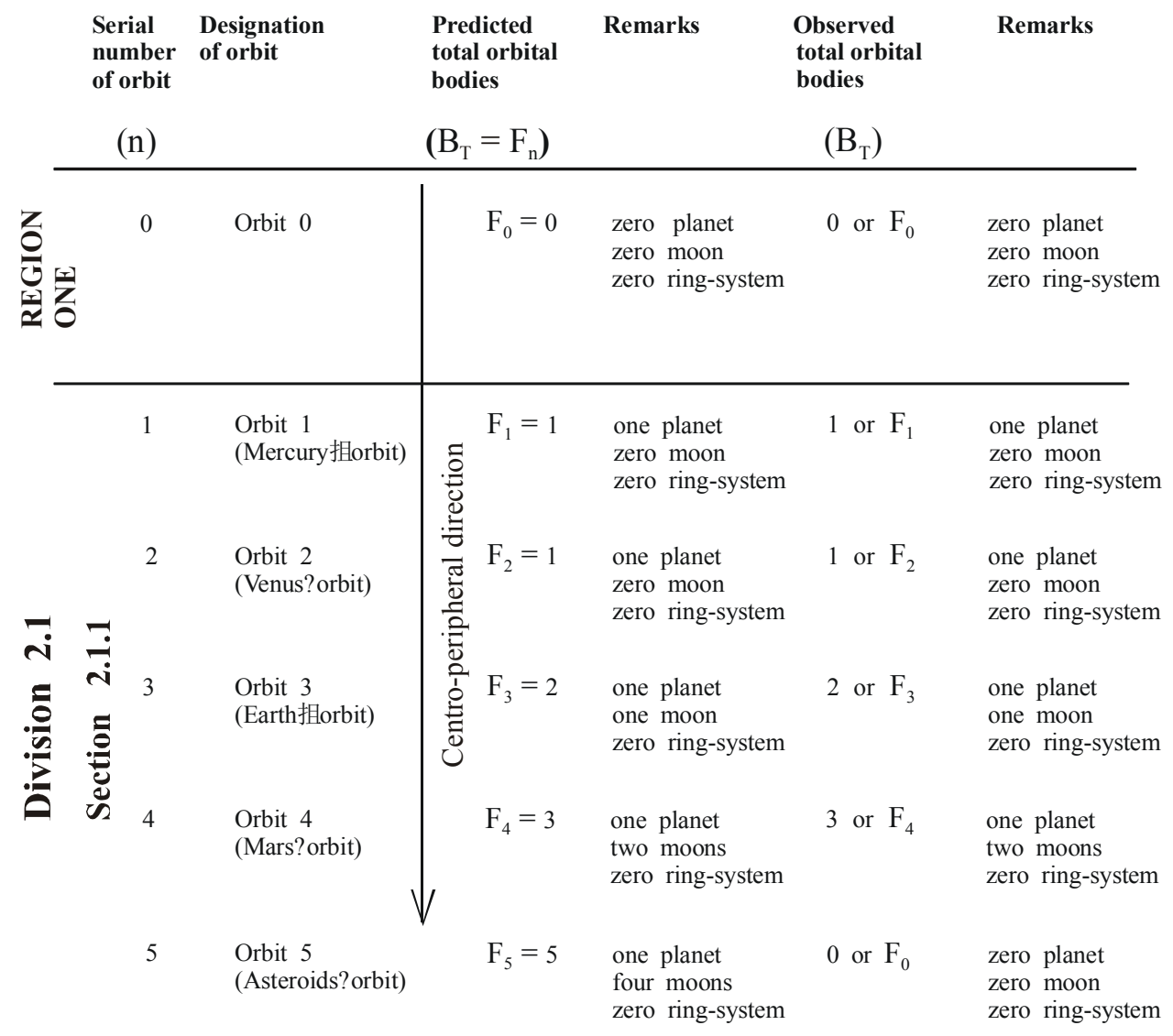

Figure 4. The predictions of the first beta total orbital bodies distribution laws. Adapted with permission [14]. 


\subsubsection{The Second Beta Total Orbital Bodies Distribution Laws:- (Law 2)}

Law 2A: In Division Zero, Section 2.2.2, Section 2.2.1, and Section 2.1.2 planetary orbits, the distribution of total orbital bodies is in the periphero-central direction.

Law 2B: In Division Zero, Section 2.2.2, Section 2.2.1, and Section 2.1.2 planetary orbits, the distribution of total orbital bodies is in Fibonacci terms.

$$
\mathrm{B}_{\mathrm{T}}=\mathrm{B}_{\mathrm{P}}+\mathrm{B}_{\mathrm{MN}}+\mathrm{B}_{\mathrm{R}}=\mathrm{F}_{3}{ }^{(13-n)}
$$

or

$$
\mathrm{B}_{\mathrm{T}}=\mathrm{F}_{3}{ }^{(13-\mathrm{n})}
$$

$\begin{array}{llllll}\begin{array}{l}\text { Serial } \\ \text { number } \\ \text { of orbit }\end{array} & \begin{array}{l}\text { Designation } \\ \text { of orbit }\end{array} & \begin{array}{l}\text { Predicted } \\ \text { total orbital } \\ \text { bodies }\end{array} & \text { Remarks } & \begin{array}{l}\text { Observed } \\ \text { total orbital } \\ \text { bodies }\end{array} & \text { Remarks }\end{array}$

(n)

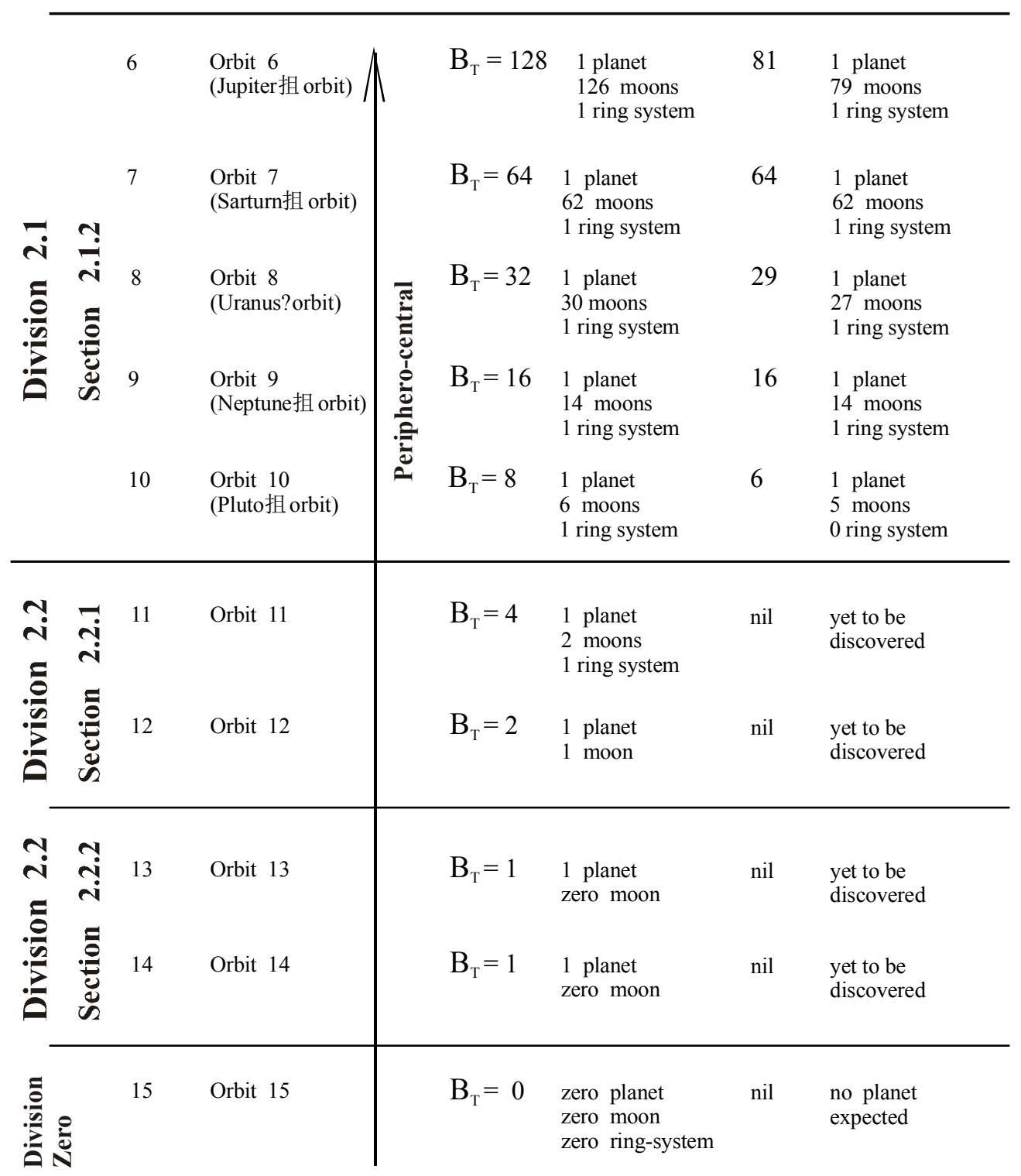

Figure 5. The predictions of the second beta total orbital bodies distribution laws for Orbit 6 to Orbit 15. Adapted and modified with permission [15].$$
\mathrm{B}_{\mathrm{T}}=\mathrm{F}_{3} \wedge\left(\mathrm{F}_{7}-\mathrm{n}\right)
$$

where $\mathrm{n}$ is the serial number of the planetary orbit

in the centro-peripheral direction

other notations remaining the same

In formulating Equations (3), (4) and (5), they were done in such a way that Orbit 6, Orbit 7, Orbit 8, Orbit 9 and Orbit 10 bear their centro-peripheral designations as shown in Figure 1, instead of their natural periphero-central designations as shown in Figure 2. The predictions of the second beta total orbital bodies distribution laws are provided in Figure 5.
\end{abstract}

total orbita 
For the second beta total orbital bodies distribution laws, only the data on Orbit 7 and Orbit 9 were employed because they appeared to be the only planetary orbits with complete data of all the Section 2.1.2 planetary orbits. "For Division Zero, Section 2.2.2, Section 2.2.1 and Section 2.1.2 planetary orbits, the beta total orbital bodies equation can be derived as follows:

Let us put the total orbital bodies equation in the form

$$
\mathrm{B}_{\mathrm{T}}=\mathrm{F}_{\mathrm{y}}^{(\mathrm{z}-\mathrm{n})}
$$

where $\mathrm{B}_{\mathrm{T}}$ is total orbital bodies

$\mathrm{F}$ is Fibonacci number

$\mathrm{n}$ is the serial number of the planetary orbit

in the centro-peripheral direction

$\mathrm{y}$ and $\mathrm{z}$ are unknown constants

The following equations for solving for $\mathrm{y}$ and $\mathrm{z}$ can be formed

$$
\begin{aligned}
& \text { From Orbit 7: } \mathrm{F}_{\mathrm{y}}{ }^{(\mathrm{z}-7)}=64 \\
& \text { From Orbit 9: } \mathrm{F}_{\mathrm{y}}{ }^{(\mathrm{z}-9)}=16 "
\end{aligned}
$$

(64 and 16 are the total orbital bodies for Orbit 7 and Orbit 9 , respectively).

When Equation 7 and Equation 8 were solved as simultaneous equations, the following solutions were obtained: $\mathrm{z}=13, \mathrm{y}=3[16,17]$

Thus, the total orbital bodies equation becomes

$$
\mathrm{B}_{\mathrm{T}}=\mathrm{F}_{3}^{(13-\mathrm{n})}
$$

For the planetary orbits in Section 2.1.2 of the solar system, predictions agreed with observations only in Orbit 7 (Saturn's orbit) and Orbit 9 (Neptune's orbit). See Figure 5.

\subsection{The Alpha Planet-Moon Bodies Distribution Laws [1]}

\subsubsection{The First Alpha Planet-Moon Bodies Distribution} Laws

The first alpha planet-moon bodies distribution laws are for Region One planetary orbit (Orbit Zero) and Section 2.1.1 planetary orbits (Orbit 1, Orbit 2, Orbit 3, Orbit 4 and Orbit 5).

The first alpha planet-moon bodies distribution laws:(Law 1)

Law IA: In Region One and Section 2.1.1 planetary orbits, the distribution of planet-moon bodies (or the distribution of planet and moons) is in the centro-peripheral direction.

Law IB: In Region One and Section 2.1.1 planetary orbits, the number of planet-moon bodies (or the number of planet and moons) in the nth planetary orbit is equal to the nth Fibonacci number, where $n$ is the serial number of the planetary orbit in the centro-peripheral direction.

$$
\mathrm{B}_{\mathrm{PM}}=\mathrm{B}_{\mathrm{P}}+\mathrm{B}_{\mathrm{Mn}}=\mathrm{F}_{\mathrm{n}}
$$

Or

$$
\mathrm{B}_{\mathrm{PM}}=\mathrm{F}_{\mathrm{n}}
$$

where $\mathrm{B}_{\mathrm{PM}}$ is number of planet-moon bodies (or number of planet and moons)

$B_{P}$ is number of planet which is usually one

$\mathrm{B}_{\mathrm{Mn}}$ is number of moons which may be zero

or a positive integer

$F_{n}$ is the nth Fibonacci number

$\mathrm{n}$ is the serial number of the planetary orbit in the centro-peripheral direction

\subsubsection{The Second Alpha Planet-Moon Bodies Distribution Laws}

The second alpha planet-moon bodies distribution laws are for Division Zero planetary orbit (Orbit 15), Section 2.2.2 planetary orbits (Orbit 14 and Orbit 13) and Section 2.2.1 planetary orbits (Orbit 12 and Orbit 11).

The second alpha planet-moon bodies distribution laws:(Law 2)

Law 2A: In Division Zero, Section 2.2.2 and Section 2.2.1 planetary orbits, the distribution of planet-moon bodies (or the distribution of planet and moons) is in the peripherocentral direction.

Law 2B: In Division Zero, Section 2.2.2 and Section 2.2.1 planetary orbits, the number of planet-moon bodies (or the number of planet and moons) in the nth planetary orbit is equal to the $n t h$ Fibonacci number, where $n$ is the serial number of the planetary orbit in the periphero-central direction.

$$
\mathrm{B}_{\mathrm{PM}}=\mathrm{B}_{\mathrm{P}}+\mathrm{B}_{\mathrm{Mn}}=\mathrm{F}_{\mathrm{n}}
$$

Or

$$
\mathrm{B}_{\mathrm{PM}}=\mathrm{F}_{\mathrm{n}}
$$

where $\mathrm{n}$ is the serial number of the planetary orbit in the periphero-central direction other notations remaining the same

It is usually convenient to put Equation (13) in such a form that the planetary orbits will retain their centro-peripheral designations.

Thus, the equation becomes

$$
\mathrm{B}_{\mathrm{PM}}=\mathrm{F}_{(15-\mathrm{n})}
$$

Or

$$
\mathrm{B}_{\mathrm{PM}}=\mathrm{F}_{(\mathrm{F} 4 \mathrm{~F} 5-\mathrm{n})}
$$

\subsubsection{The Third Alpha Planet-Moon Bodies Distribution Laws}

The third alpha planet-moon bodies distribution laws are for Section 2.1.2 planetary orbits (Orbit 10, Orbit 9, Orbit 8, Orbit 7 and Orbit 6).

The third alpha planet-moon bodies distribution laws:(Law 3)

Law 3A: In Section 2.1.2 planetary orbits, the distribution of planet-moon bodies (or the distribution of planet and moons) is in the periphero-central direction.

Law 3B: In Section 2.1.2 planetary orbits, the distribution 
of planet-moon bodies (or the distribution of planet and moons) is in Fibonacci terms.

$$
\mathrm{B}_{\mathrm{PM}}=\mathrm{B}_{\mathrm{P}}+\mathrm{B}_{\mathrm{Mn}}=\mathrm{F}_{(15-\mathrm{n})}+5 \mathrm{~F}_{(20-2 \mathrm{n})}+\mathrm{F}_{3}
$$

or

$$
\mathrm{B}_{\mathrm{PM}}=\mathrm{F}_{(15-\mathrm{n})}+5 \mathrm{~F}_{(20-2 \mathrm{n})}+\mathrm{F}_{3}
$$

or

$$
\mathrm{B}_{\mathrm{PM}}=\mathrm{F}_{(\mathrm{F} 4 \mathrm{F5}-\mathrm{n})}+\mathrm{F}_{5} \mathrm{~F}_{(\mathrm{FSF3F3}-\mathrm{nF3})}+\mathrm{F}_{3}
$$

where $\mathrm{n}$ is the serial number of the planetary orbit

in the centro-peripheral direction

Other notations remaining the same

In formulating Equations (16), (17) and (18), they were done in such a way that Orbit 6 , Orbit 7, Orbit 8, Orbit 9 and Orbit 10 are with their centro-peripheral designations, instead of the natural periphero-central designations.
The formulation of the alpha planet-moon bodies distribution laws is cumbersome and complicated when compared with that of the beta total orbital bodies distribution laws. It cannot be presented here because of constraint of space. However, the details can be found elsewhere [1].

\section{Results (The Predictions of Chime's Laws for Distribution of Bodies)}

In Table 2, the predictions of the alpha planet-moon bodies distribution laws and beta total orbital bodies distribution laws are compared with the observed data, for Orbit zero to Orbit 15.

The predicted planetary ring-systems in column 3 were

\begin{tabular}{|c|c|c|c|c|c|c|}
\hline S/No & Orbit & $\begin{array}{l}\text { Number of } \\
\text { Planetary ring- } \\
\text { system }\end{array}$ & $\begin{array}{l}\text { Prediction } \\
\text { of } \alpha \mathrm{PMB} \\
\text { equations }\end{array}$ & $\begin{array}{l}\text { Prediction } \\
\text { of } \beta \text { TOB } \\
\text { equations }\end{array}$ & Observation & Remarks \\
\hline 0 & Orbit zero & 0 (predicted) & $\begin{array}{l}0 \text { PMB: } \\
0 \text { planet } \\
0 \text { moon }\end{array}$ & $\begin{array}{l}0 \text { TOB: } \\
0 \text { planet } \\
0 \text { moon } \\
0 \text { ring-system }\end{array}$ & $\begin{array}{l}0 \text { TOB: } \\
0 \text { planet } \\
0 \text { moon } \\
0 \text { ring-system }\end{array}$ & $\begin{array}{l}\text { Observation } \\
\text { agreed with } \\
\text { prediction }\end{array}$ \\
\hline 1 & $\begin{array}{l}\text { Orbit } 1 \\
\text { (Mercury's } \\
\text { orbit) }\end{array}$ & 0 (discovered) & $\begin{array}{l}1 \mathrm{PMB}: \\
1 \text { planet } \\
0 \text { moon }\end{array}$ & $\begin{array}{l}1 \text { TOB: } \\
1 \text { planet } \\
0 \text { moon } \\
0 \text { ring-system }\end{array}$ & $\begin{array}{l}1 \text { TOB: } \\
1 \text { planet } \\
0 \text { moon } \\
0 \text { ring-system }\end{array}$ & $\begin{array}{l}\text { Observation } \\
\text { agreed with } \\
\text { prediction }\end{array}$ \\
\hline 2 & $\begin{array}{l}\text { Orbit } 2 \\
\text { (Venus' orbit) }\end{array}$ & 0 (discovered) & $\begin{array}{l}1 \text { PMB: } \\
1 \text { planet } \\
0 \text { moon }\end{array}$ & $\begin{array}{l}1 \text { TOB: } \\
1 \text { planet } \\
0 \text { moon } \\
0 \text { ring-system }\end{array}$ & $\begin{array}{l}1 \text { TOB: } \\
1 \text { planet } \\
0 \text { moon } \\
0 \text { ring-system }\end{array}$ & $\begin{array}{l}\text { Observation } \\
\text { agreed with } \\
\text { prediction }\end{array}$ \\
\hline 3 & $\begin{array}{l}\text { Orbit } 3 \\
\text { (Earth's orbit) }\end{array}$ & 0 (discovered) & $\begin{array}{l}2 \text { PMBs: } \\
1 \text { planet } \\
1 \text { moon }\end{array}$ & $\begin{array}{l}2 \text { TOBs: } \\
1 \text { planet } \\
1 \text { moon } \\
0 \text { ring-system }\end{array}$ & $\begin{array}{l}2 \text { TOBs: } \\
1 \text { planet } \\
1 \text { moon } \\
0 \text { ring-system }\end{array}$ & $\begin{array}{l}\text { Observation } \\
\text { agreed with } \\
\text { prediction }\end{array}$ \\
\hline 4 & $\begin{array}{l}\text { Orbit } 4 \\
\text { (Mars' orbit) }\end{array}$ & 0 (discovered) & $\begin{array}{l}3 \text { PMBs: } \\
1 \text { planet } \\
2 \text { moons }\end{array}$ & $\begin{array}{l}\text { 3TOBs: } \\
1 \text { planet } \\
2 \text { moons }\end{array}$ & $\begin{array}{l}\text { 3TOBs: } \\
1 \text { planet } \\
2 \text { moons }\end{array}$ & $\begin{array}{l}\text { Observation } \\
\text { agreed with } \\
\text { prediction }\end{array}$ \\
\hline 5 & $\begin{array}{l}\text { Orbit } 5 \\
\text { (Asteroids' } \\
\text { orbit) }\end{array}$ & 0 (discovered) & $\begin{array}{l}5 \text { PMBs: } \\
1 \text { planet } \\
4 \text { moons }\end{array}$ & $\begin{array}{l}5 \text { TOBs: } \\
1 \text { planet } \\
4 \text { moons } \\
0 \text { ring-system }\end{array}$ & $\begin{array}{l}0 \text { TOBs: } \\
0 \text { planet } \\
0 \text { moons } \\
0 \text { ring-system }\end{array}$ & $\begin{array}{l}1 \text { planet } \\
4 \text { moons } \\
\text { missing }\end{array}$ \\
\hline 6 & $\begin{array}{l}\text { Orbit } 6 \\
\text { (Jupiter' orbit) }\end{array}$ & 1 (discovered) & $\begin{array}{l}141 \text { PMBs: } \\
1 \text { planet } \\
140 \text { moons }\end{array}$ & $\begin{array}{l}128 \text { TOBs: } \\
1 \text { planet } \\
126 \text { moons } \\
1 \text { ring-system }\end{array}$ & $\begin{array}{l}81 \text { TOBs: } \\
1 \text { planet } \\
79 \text { moons } \\
1 \text { ring-system }\end{array}$ & $\begin{array}{l}61 \text { or } 47 \text { moons } \\
\text { missing }\end{array}$ \\
\hline
\end{tabular}
done with regression equations $[18,19]$.

Table 2. Predictions compared with observations for Orbit zero to Orbit 15. Adapted with permission [20]. 
Table 2. Continued.

\begin{tabular}{|c|c|c|c|c|c|c|}
\hline S/No & Orbit & $\begin{array}{l}\text { Number of } \\
\text { Planetary ring-system }\end{array}$ & $\begin{array}{l}\text { Prediction } \\
\text { of } \alpha \text { PMB } \\
\text { equations }\end{array}$ & $\begin{array}{l}\text { Prediction } \\
\text { of } \beta \text { TOB } \\
\text { equations }\end{array}$ & Observation & Remarks \\
\hline 7 & $\begin{array}{l}\text { Orbit } 7 \\
\text { (Saturn' orbit) }\end{array}$ & 1 (discovered) & $\begin{array}{l}63 \text { PMBs: } \\
1 \text { planet } \\
62 \text { moons }\end{array}$ & $\begin{array}{l}64 \text { TOBs: } \\
1 \text { planet } \\
62 \text { moons } \\
1 \text { ring-system }\end{array}$ & $\begin{array}{l}64 \text { TOBs: } \\
1 \text { planet } \\
62 \text { moons } \\
1 \text { ring-system }\end{array}$ & $\begin{array}{l}\text { Observation } \\
\text { agreed with } \\
\text { prediction }\end{array}$ \\
\hline 8 & $\begin{array}{l}\text { Orbit } 8 \\
\text { (Uranus' orbit) }\end{array}$ & 1 (discovered) & $\begin{array}{l}30 \text { PMBs: } \\
1 \text { planet } \\
29 \text { moons }\end{array}$ & $\begin{array}{l}32 \text { TOBs: } \\
1 \text { planet } \\
30 \text { moons } \\
1 \text { ring-system }\end{array}$ & $\begin{array}{l}29 \text { TOBs: } \\
1 \text { planet } \\
27 \text { moons } \\
1 \text { ring-system }\end{array}$ & $\begin{array}{l}2 \text { or } 3 \text { moons } \\
\text { missing }\end{array}$ \\
\hline 9 & $\begin{array}{l}\text { Orbit } 9 \\
\text { (Neptune's } \\
\text { orbit) }\end{array}$ & 1 (discovered) & $\begin{array}{l}15 \text { PMBs: } \\
1 \text { planet } \\
14 \text { moons }\end{array}$ & $\begin{array}{l}16 \text { TOBs: } \\
1 \text { planet } \\
14 \text { moons } \\
1 \text { ring-system }\end{array}$ & $\begin{array}{l}16 \text { TOBs: } \\
1 \text { planet } \\
14 \text { moons } \\
1 \text { ring-system }\end{array}$ & $\begin{array}{l}\text { Observation } \\
\text { agreed with } \\
\text { Prediction }\end{array}$ \\
\hline 10 & $\begin{array}{l}\text { Orbit } 10 \\
\text { (Pluto's orbit) }\end{array}$ & 1 (predicted) & $\begin{array}{l}7 \text { PMBs: } \\
1 \text { planet } \\
6 \text { moons }\end{array}$ & $\begin{array}{l}8 \text { TOBs: } \\
1 \text { planet } \\
6 \text { moons } \\
1 \text { ring-system }\end{array}$ & $\begin{array}{l}6 \text { TOBs: } \\
1 \text { planet } \\
5 \text { moons } \\
0 \text { ring-system }\end{array}$ & $\begin{array}{l}1 \text { moon } \\
\text { missing } \\
1 \text { ring-system } \\
\text { missing }\end{array}$ \\
\hline 11 & Orbit 11 & 1 (predicted) & $\begin{array}{l}3 \text { PMBs: } \\
1 \text { planet } \\
2 \text { moons }\end{array}$ & $\begin{array}{l}4 \text { TOBs: } \\
1 \text { planet } \\
2 \text { moons } \\
1 \text { ring-system }\end{array}$ & Not yet discovered & $\begin{array}{l}\text { Not yet } \\
\text { discovered }\end{array}$ \\
\hline 12 & Orbit 12 & 0 (predicted) & $\begin{array}{l}2 \text { PMBs: } \\
1 \text { planet } \\
1 \text { moon }\end{array}$ & $\begin{array}{l}2 \text { TOBs: } \\
1 \text { planet } \\
1 \text { moon } \\
0 \text { ring-system }\end{array}$ & Not yet discovered & $\begin{array}{l}\text { Not yet } \\
\text { discovered }\end{array}$ \\
\hline 13 & Orbit 13 & 0 (predicted) & $\begin{array}{l}1 \text { PMB: } \\
1 \text { planet } \\
0 \text { moon }\end{array}$ & $\begin{array}{l}1 \text { TOBs: } \\
1 \text { planet } \\
0 \text { moon } \\
0 \text { ring-system }\end{array}$ & Not yet discovered & $\begin{array}{l}\text { Not yet } \\
\text { discovered }\end{array}$ \\
\hline 14 & Orbit 14 & 0 (predicted) & $\begin{array}{l}1 \text { PMB: } \\
1 \text { planet } \\
0 \text { moon }\end{array}$ & $\begin{array}{l}1 \text { TOBs: } \\
1 \text { planet } \\
0 \text { moon } \\
0 \text { ring-system }\end{array}$ & Not yet discovered & $\begin{array}{l}\text { Not yet } \\
\text { discovered }\end{array}$ \\
\hline 15 & Orbit 15 & 0 (predicted) & $\begin{array}{l}0 \text { PMB: } \\
0 \text { planet } \\
0 \text { moon }\end{array}$ & $\begin{array}{l}0 \text { TOBs: } \\
0 \text { planet } \\
0 \text { moon } \\
0 \text { ring-system }\end{array}$ & Not yet discovered & $\begin{array}{l}\text { No planet } \\
\text { expected in this } \\
\text { location }\end{array}$ \\
\hline
\end{tabular}

$\alpha$ PMB means alpha planet-moon body. PMBs means planet-moon bodies. $\beta$ TOB means beta total orbital body. TOBs means total orbital bodies.

\section{Discussions}

\subsection{Either the Alpha Group of Laws or the Beta Group of Laws Are Operative in the Solar System}

The predicted number of moons by the alpha group of laws and by the beta group of laws for each of the 14 planetary orbits of the solar system was the same except in Orbit 6 (Jupiter's orbit) and Orbit 8(Saturn's orbit). This suggests that it is either the alpha planet-moon bodies distribution laws or the beta total orbital bodies distribution laws that are operative in the solar system, but not both of them. The beta total orbital bodies distribution laws are simpler, more elegant and more Fibonacci-friendly than the alpha planetmoon bodies distribution laws (or the alpha total orbital bodies distribution laws). For the beta total orbital bodies distribution laws, a set of two laws was required to predict the 14-orbit solar system but for the alpha planet-moon bodies distribution laws, a set of three laws was required to predict the 14-orbit solar system. Occam's razor would 
therefore favour the beta total orbital bodies distribution laws rather than the alpha planet-moon bodies distribution laws or the alpha total orbital bodies distribution laws.

\subsection{The Distribution of Planets, Moons and Planetary Ring-System Is Non-Random}

If the beta total orbital bodies distribution laws are operative in the solar system, it would mean that the orbital distribution of planets, moons and planetary ring-system in the solar system is non-random. If the alpha planet-moon bodies distribution laws are operative in the solar system, it would mean that the orbital distribution of planets and moons in the solar system is non-random. Moreover, a different analysis had already shown that the distribution of planetary ring-system in the solar system is non-random $[18,19]$.

\subsection{The Solar System is a 14-Orbit System}

The alpha planet-moon bodies distribution laws and the beta total orbital bodies distribution laws each predicted a 16orbit architectural system. A 16-orbit architectural system is consistent with a 14 orbit solar system, meaning that the solar system has 14 planetary orbits. Orbit 1 to Orbit 14 plus the two Orbit Zeros made up the 16-orbit architectural system (Figure 2).

\subsection{Zero Planet Was Predicted for Orbit Zero}

Orbit Zero is located in the center of the solar system, which can be taken to be located in the center of the Sun. Zero planet was predicted for Orbit Zero. One does not expect any planet to exist in that location.

\subsection{The Section 2.1.1 Planets}

\subsubsection{Mercury Is the First Planet of the Solar System}

Mercury is in Orbit One, meaning that Mercury is the first planet of the solar system. Therefore, Le Verrie's Vulcan does not exist. Le Verrie's prediction was that Vulcan should be located between the Sun and Mercury. Although unsuccessful searches for Vulcan had been made in the past, the theoretical finding in this study is strong theoretical evidence that Vulcan does not exist.

Also, there is no theoretical evidence that Mercury has any moon or a ring-system. Physical exploration has not detected any moon around Mercury [21].

\subsubsection{Venus Has no Moon and Has no Ring-System}

There is no theoretical evidence that Venus has any moon or a ring-system. Moreover, physical exploration has not detected any moon around Venus [22], and no standard ringsystem has been detected around Venus [23].

\subsubsection{Earth Has no Additional Moon and Has no Ring-System}

There is no theoretical evidence that Earth has an additional moon or a ring-system. Tombaugh was one of those who made unsuccessful searches for additional moons of the Earth. Others who claimed to have discovered additional Earth's moons in the past did not have their claims authenticated.

\subsubsection{Mars Has no Additional Moon and Has no Ring-System}

There is no theoretical evidence that Mars has an additional moon or a ring-system. Moreover, physical exploration has not detected any additional moon around Mars [24, 25] and no standard ring-system has been detected around Mars [25].

\subsubsection{Planet 5 Has 4 Moons and Ceres Is Not Planet 5}

Planet 5, the Orbit 5 planet which could not form, was described as a ghost planet [26]. Planet 5 has 4 ghost moons. The Orbit 5 asteroids are the physical remains of the ghost Planet 5 and the 4 ghost moons of Planet 5, although the asteroids has suffered depletion, as only about three-quarters of the original mass is remaining [27]. (Estimated initial total orbital mass of Orbit $5=3.0 \times 10^{21} \mathrm{~kg}$, total mass of all the bodies presently in Orbit $5=2.3 \times 10^{21} \mathrm{~kg}$ ) [27]. Ceres is not Planet 5 because (a) Ceres has a mass of $9.39 \times 10^{20} \mathrm{~kg}$ which is less than the estimated mass of Planet 5 which is 2.9 x $10^{21} \mathrm{~kg}$ [26]. (b) Ceres has no moon but Planet 5 has 4 moons [26]. Ceres is an asteroid. ("An asteroid is a major compact body in a primary star-centric orbit which is not a planet)" [11].

\subsection{The Section 2.1.2 Planets}

\subsubsection{Many Jupiter's Moons Are Missing}

For Orbit 6 (Jupiter' orbit), the alpha planet-moon bodies distribution laws predicted 140 moons while the beta total orbital bodies distribution laws predicted 126 moons. So far, only 79 Jupiter's moons have been discovered. Thus, whichever group of bodies distribution laws is operative in the solar system, the prediction is that many Jupiter's moons are missing. These have been labeled the missing moons of Jupiter $[10,26]$, and they are likely to exist as asteroids. The actual number of missing moons of Jupiter will depend on which bodies distribution laws are operative in the solar system, as well as on the number of Jupiter's moons that may be discovered in future. Based on the currently available data, it is either 47 moons (beta total orbital bodies distribution laws prediction) or 61 moons (alpha planet-moon bodies distribution laws prediction) of Jupiter that are missing.

\subsubsection{All the Saturn's Moons Have Been Discovered}

Saturn has 62 discovered moons which agreed with the theoretical predictions. This suggests that all the Saturn's moons have been discovered. In its final combing of Saturn's neighbourhood, Cassini did not detect any other moon around Saturn.

\subsubsection{Some of Uranus' Moons Are Undiscovered}

For Orbit 8 (Uranus' orbit), the alpha planet-moon bodies distribution laws predicted 29 moons while the beta total orbital bodies distribution laws predicted 30 moons. So far, only 27 moons have been discovered. Thus, if only 2 more 
moons of Uranus can be discovered, it would mean that the alpha planet-moon bodies distribution laws are operative in the solar system and that the solar system is an alpha solar system in which alpha laws are operative. For identification purposes, the two moons have been labeled Ezechi and Akanene in the order in which they will be discovered [26]. However, if only 3 more moons of Uranus can be discovered, it would mean that the beta total orbital bodies distribution laws are operative in the solar system and that the solar system is a beta solar system in which beta laws are operative. For identification purposes, the three moons have been labeled Ezechi, Akanene and Ikechukwu in the order in which they will be discovered [26]. Because of the important role of Orbit 8 (Uranus' orbit) in determining which group of bodies distribution laws is operative in the solar system, Orbit 8 (Uranus' orbit) was labeled the crucial planetary orbit and Uranus was labeled the crucial planet [26].

\subsubsection{All the Neptune's Moons Have Been Discovered}

Neptune has 14 discovered moons which agreed with the theoretical predictions. This means that all the Neptune's moons have been discovered and none of Neptune's moons is missing.

\subsubsection{Pluto Has 6 Moons and a Ring-System}

Six moons as well as a ring-system was predicted for Pluto. So far, only 5 of the 6 Pluto's moons have been discovered. The possible fate of Pluto's 6th moon was discussed in detail elsewhere [28]. For purposes of identification, the 6th moon of Pluto was labeled Warsawlene [26]. That name was based on the belief that Warsawlene saw war like Warsaw [28]. There was a strong suspicion that Warsawlene did not survive the battle of Neplu [28]. As regards the ring-system predicted for Pluto, the New Horizon did not detect any standard ring-system around Pluto during its flyby of Pluto in July, 2015. It is possible that Pluto's relative small mass could not provide enough gravitational force that will prevent the ring-system from dissipating.

\subsubsection{Pluto Is Planet 10}

Pluto was predicted to have 6 moons and 5 out of the predicted 6 moons have been discovered already. Pluto is the principal body of Orbit 10, because not only that Pluto has the largest mass of all the bodies in Orbit 10, other orbital bodies of
Orbit 10 are in orbit around Pluto. By our working definition of planet $[11,12]$, Pluto is a planet. It is also important to note that none of Neptune's moons is missing as all the 14 moons predicted for Neptune have been discovered. Therefore, Pluto is not an escaped moon of Neptune as was first suggested by Lyttleton in 1936 [5]. Pluto is Planet 10.

\subsection{Division 2.2 of the Solar System}

\subsubsection{Planet Eleven Has Not Yet Been Discovered}

Planet Eleven is the first planet in Division 2.2 of the solar system. Planet Eleven, the next planet after Pluto, was predicted to have 2 moons and a ring-system. In an earlier study, Planet Eleven was predicted to have a mass of $2.4 \mathrm{x}$ $10^{18} \mathrm{~kg}$ and a combined mass of $6.7 \times 10^{17} \mathrm{~kg}$ for its moons [29]. In the same study, the estimated semimajor axis and eccentricity of Planet Eleven's orbit were 46AU and 0.79 respectively [29]. None of the bodies discovered so far in the solar system matched that description $[29,30]$.

\subsubsection{Other Division 2.2 Planets}

The parameters of the other planetary orbits and planets in Division 2.2 of the solar system have not been worked out out to reasonable levels.

\subsection{The Number of Planets, Moons and planetary Ring-Systems in the Solar System}

\subsubsection{There Are Between 9 and 13 Formed Planets in the Solar System}

Fourteen planetary orbits were predicted for the solar system. Nine planets have already been discovered in the first ten planetary orbits. The number of planets that can be discovered in the remaining four planetary orbits is between zero and four. Thus, there are between 9 and 13 formed planets in the solar system. Table 3 illustrates the different options for the number of formed planets and number of ghost planets in the Solar System [30]. Table 4 illustrates the sectional distribution of planets in the solar system. Table 3 is based on the fact that the number of formed planets plus the number of ghost planets equals 14 (Equation 19) [30].

Number of formed planets + number of ghost planets $=14$

Table 3. The different options for the number of planets in the Solar System. Adapted with permission [30]

\begin{tabular}{lllll}
\hline & Option A & Option B & Option C & Option D \\
\hline Number of formed planets & 9 & 10 & 11 & 12 \\
Number of ghost planets & 5 & 4 & 3 & 13 \\
Total number Of planets & 14 & 14 & 14 & 1 \\
\hline
\end{tabular}

Ghost planets are likely to exist as asteroids.

Table 4. The sectional distribution of planets in the solar system. Adapted with permission [30]

\begin{tabular}{llll}
\hline Section & Number of formed planets & Number of ghost planets & Total numberOf planets \\
\hline Section 2.1 .1 & 4 & 1 & 5 \\
Section 2.1 .2 & 5 & 0 & 5 \\
Section 2.2 .1 & $0-2$ & $2-0$ & 2 \\
Section 2.2 .2 & $0-2$ & $2-0$ & 2 \\
Total & $9-13$ & $5-1$ & 14 \\
\hline
\end{tabular}

Ghost planets are likely to exist as asteroids. 


\subsubsection{One Hundred and Ninety Moons Discovered so Far Out of the Predicted 248 Moons}

As regards the total number of moons in the solar system, the alpha planet-moon bodies distribution laws predicted 261 moons, while the beta total orbital bodies distribution laws predicted 248 moons [31]. However, only 190 moons have been discovered so far. Four moons did not form in Orbit 5. The fates of the predicted 2 moons in Orbit 11 and one moon in Orbit 12 are unknown. Most of the missing moons are in Orbit 6 (Jupiter's orbit). Ghost moons are likely to exist as asteroids. The total number of moons that will eventually be discovered in the solar system will depend on the number of moons that will be discovered in Orbit 6 eventually. It will also depend on whether Planet Eleven's moons and Planet Twelve's moon will be found intact.

\subsubsection{There Are 6 Planetary Ring-Systems in the Solar System [18, 19]}

Pluto (Planet 10) and Planet 11 were predicted to have ring-systems $[18,19]$. These are in addition to the 4 ringsystems discovered around Jupiter, Saturn, Neptune and Uranus.

\subsection{Other Findings Regarding the Planets, Moons and Planetary Ring-Systems}

\subsubsection{Every Planetary Orbit Has a Planet}

The prediction of the alpha group and the beta group of laws is that every planetary orbit has a planet. At a glance, this does not appear to be true. For example, in Orbit 5, the asteroids' orbit, there is no formed planet in existence. However, this does not violate the laws for distribution of bodies. In any planetary orbit in which a planet did not form to conclusion, the matter which would have formed the planet is expected to end up as asteroids as happened in Orbit 5 of the solar system. Also, as happened in Orbit 5, this matter could suffer from depletion [27]. The possible existence of holes laws has also been mentioned elsewhere [32]. Holes laws are expected to determine or describe the planetary orbits in the solar system in which matter meant to form planets and/or moons ended up as asteroids [32]. In conclusion, therefore, every planetary orbit has a planet. However, the planet can exist either as a formed planet or as a ghost planet (of which asteroids are its physical remains).

\subsubsection{None of the Discovered Planets in the Solar System Has Surplus Moon}

None of the discovered planets in the solar system has surplus moon. In a situation in which the distribution of moon is non-random, a surplus moon of a planet may suggest asteroid capture by the planet or capture of another planet's moon by the planet. Some of the planets have the number of moons predicted by theory, while some others have the number of moons which is less than that predicted by theory. Absence of theoretical evidence of asteroid capture by any of the known planets of the solar system should be a major setback for those hypotheses or theories which claimed that some of the moons of the solar planets are captured asteroids. Examples include the two moons of Mars (Phobos and Deimos) and some of the moons of Neptune.

\subsubsection{A Good Theory on the Formation of Planets and Moons Should Be Based on a Process that Is not Prone to Chance}

The laws for the distribution of bodies have shown that the orbital distribution of planets, moons and planetary ringsystems in the solar system is nonrandom. It was shown in an earlier study that their distribution by mass is nonrandom in the solar system [33]. Therefore, any good theory on the formation of planets and moons in the solar system should be based on a process that is not prone to chance. Of all the proposed hypotheses or theories on the formation of planets and moons in the solar system, the only one that appears not to be prone to chance is co-formation or co-accretion. "It is only in co-formation or co-accretion that appropriate amount of matter could be at the appropriate orbits at the appropriate time to form appropriate number of bodies of appropriate masses" [34].

\subsubsection{A Good Theory on the Formation of Planetary Ring-Systems Should Be Based on a Process That Is Not Prone to Chance}

As already mentioned in 4.9.3 above, the distribution of planetary ring-systems in the solar system has been shown to be non-random both by number and by mass. Also, of interest is the fact that the planetary ring-systems behaved as single bodies during numerical strength analyses [18] even though the ring-system does not rotate as a rigid body. Some astronomers think that destruction of satellites which came within the Roche's limit of their respective planets may be a source of material for the ring-system's formation or sustenance, yet we saw no strong theoretical evidence of loss of moons by any of the planets with ring-systems, with the exception of Jupiter which is a special case. Saturn which has the biggest ring-system in the solar system has no missing moon. In the case of Uranus, there is no reason why the undiscovered two or three moons cannot be found, Therefore, any good theory on the formation of planetary ring-systems in the solar system should be based on a process that is not prone to chance. The theory on the formation of planetary ring-systems should also have a common application to all the planets with ring-systems. As a possible method of formation of planetary ring-systems, suggested methods include co-formation in which the ring-system formed along with the planet and moons, or delayed co-formation in which the ring-system formed after the planet and moons had formed [35].

\subsection{The 2006 Definition of Planet by IAU Has Serious Flaws}

The definition of planet adopted by the International Astronomical Union(IAU) in 2006 has a serious flaw as it was unable to recognize that Pluto is a planet. That was 
because of one of its requirements that a celestial body should clear its neighborhood for it to qualify as a planet [36]. Another flaw in the IAU definition of planet is that it has no inherent limit regarding the number of planets in our solar system. It is an open cheque. We saw in this article that the distribution of planets, moons and planetary ring-system in the solar system is systematic and predictable, and there is a limit to the possible number of planets in the solar system. Yet another flaw in the IAU definition of planet is that, by limiting the definition of planet to the solar system, it gives a false (although unintended) impression that planets exist only in our solar system. Such serious flaws in the IAU definition of planet warrant a radical revision of the definition of planet. A new and better definition of planet has become necessary. Previous workers had highlighted this problem and had also called for revision of the definition of planet [12, 37, 38].

\section{Conclusions}

(1) This study critically examined the predictions of Chime's laws for distribution of bodies in order to see if the distribution of planets, moons and planetary ring-systems in the solar system is random or non-random. The study has shown that planets, moons and planetary ring-systems are non-randomly distributed in the solar system. However, one problem that arose from this study is determining whether it is the alpha planet-moon bodies distribution laws or the beta total orbital bodies distribution laws that are operative in the solar system. Although the simplicity, elegance and Fibonacci-friendliness of the beta total orbital bodies distribution laws make them the more likely candidates, the ultimate test would be to see if two or three more moons will be discovered around Uranus.

(2) Another important thing that is obvious from this study is that everything we know about the solar system so far concerns only Division 2.1 of the solar system, i.e., Orbit One to Orbit Ten, with their planets, moons and planetary ring-systems. Presently, nothing is known about the predicted Division 2.2 of the solar system which comprises Orbit Eleven to Orbit Fourteen. For this virgin division, systematic theoretical explorations are necessary before any meaningful physical exploration could commence. And it may be wise to start with Orbit Eleven and its planet.

(3) As regards Division 2.1 of the solar system, there is no theoretical reason why any standard ring-system or any more moon should be expected to be discovered in Section 2.1.1 of the solar system. It is advisable therefore, for astronomers to channel their energies and limited resources towards Section 2.1.2 of the solar system, specifically to Orbit 6 (Jupiter's orbit), Orbit 8 (Uranus' orbit) Orbit 9 (Neptune's orbit) and Orbit 10 (Pluto's orbit), where it is necessary to resolve some of the new puzzles generated by this study. (a) How many Jupiter's moons are actually missing? (b) Which group of laws is operative in the solar system? (c) Where is Warsawlene, the predicted sixth moon of Pluto? Was it captured by Neptune, is it hiding somewhere in Pluto's neighbourhood or was it destroyed during its development, making it to now exist as asteroids?

(4) This study has exposed some very serious flaws in the 2006 IAU definition of planet. Because of these flaws, the 2006 IAU definition of planet was not able to recognize that Pluto is a planet. These problems deserve urgent attention.

\section{Recommendations}

(1) There should be a systematic search for the undiscovered moons of Uranus, namely, Ezechi, Akanene and possibly Ikechukwu, using an orbiter. Their discovery will corroborate the theoretical finding that it is the beta total orbital bodies distribution laws that are operative in the solar system.

(2) There should be a systematic search for the sixth moon of Pluto, Warsawlene, in both Neptune's territory and Pluto's territory, using different orbiters. Although, the chances of seeing Warsawlene intact are very slim, confirmation of the presence of non-intact Warsawlene is very vital to The Theory Of Universality because that is what The Theory of Universality is going to predict.

(3) The planetary status of Pluto should be recognized. It is a right and not a privilege. The planetary status of Pluto does not require any proclamation or declaration by anybody or any institution. The planetary status of Pluto is based on well established scientific facts.

(4) Serious and concerted efforts should be made to discover Planet Eleven as soon as possible.

(5) Serious and systematic theoretical explorations of Division 2.2 of the solar system should commence immediately as a prelude to future physical explorations of this virgin division.

(6) A new and better definition of planet has become necessary.

\section{Disclosure of Financial Support}

This study did not receive any financial support.

\section{Conflict of Interest}

The author does not have any possible conflict of interest.

\section{References}

[1] P. E. Chime, Chap 14 The Laws For The Distribution Of Peripheral Bodies In The Solar System. In The Number And Distribution Of Planetary Orbits, Planets, Moons And Planetary Ring-Systems In The Solar System (Charles-Monica Press, Enugu, 2015) p $127-136$.

[2] G. A. Landis, Origin of Martian Moons from Binary Asteroid Dissociation. American Association for the Advancement of Science Annual Meeting; Boston, MA, 2001.

[3] Robin Canup, Julien Salmon, Origin of Phobos and Deimos by the impact of a Vesta-to-Ceres sized body with Mars. Science Advances 18 Apr 2018: Vol. 4, no. 4, eaar6887 DOI: $10.1126 /$ sciadv.aar6887 
[4] R. M. Canup, E. Asphaug, Origin of the Moon in a giant impact near the end of the Earth's formation. Nature (2001) 412: 708-712

[5] R. A. Lyttleton, On the possible results of an encounter of Pluto with the Neptunian system. Monthly Notices of the Royal Astronomical Society (1936) Vol. 97, p. 108- 115.

[6] P. E. Chime, Chap 5 The Proposed Regions, Divisions, And Sections Of The Solar System. In The Planetary Orbits And Matter Of The Solar System: Analysis 1 (Charles-Monica Press, Enugu, 2012) p 40 - 44.

[7] P. E. Chime, The Number And Distribution Of Planetary Orbits, Planets, Moons And Planetary Ring-Systems In The Solar System, (Charles-Monica Press, Enugu, 2015) Appendix B, Figure B1, p 289.

[8] P. E. Chime, The Number And Distribution Of Planetary Orbits, Planets, Moons And Planetary Ring-Systems In The Solar System, (Charles-Monica Press, Enugu, 2015) Appendix B, Figure B2, p 290.

[9] P. E. Chime, The Number And Distribution Of Planetary Orbits, Planets, Moons And Planetary Ring-Systems In The Solar System, (Charles-Monica Press, Enugu, 2015) Appendix $\mathrm{J}$, Figure J1, p 304.

[10] P. E. Chime, Chap 11 The Planet-Moon Bodies: Examination Of Different Architectural Models For The Solar System. In The Number And Distribution Of Planetary Orbits, Planets, Moons And Planetary Ring-Systems In The Solar System (Charles-Monica Press, Enugu, 2015) p 63 - 90.

[11] P. E. Chime, Chap 4 The Structure Of The Solar System. In The Number And Distribution Of Planetary Orbits, Planets, Moons And Planetary Ring-Systems In The Solar System (Charles-Monica Press, Enugu, 2015) p 24 - 31.

[12] P. E. Chime, Chap 23. Definition Of Planet. In The Planetary Orbits And Matter Of The Solar System: Analysis 1 (CharlesMonica Press, Enugu, 2012) p 227 - 232.

[13] P. E. Chime, The Number And Distribution Of Planetary Orbits, Planets, Moons And Planetary Ring-Systems In The Solar System, (Charles-Monica Press, Enugu, 2015) Appendix C, Figure C1, p 291

[14] P. E. Chime, The Number And Distribution Of Planetary Orbits, Planets, Moons And Planetary Ring-Systems In The Solar System, (Charles-Monica Press, Enugu, 2015) Appendix $\mathrm{H}$, Figure H1, p 300.

[15] P. E. Chime, The Number And Distribution Of Planetary Orbits, Planets, Moons And Planetary Ring-Systems In The Solar System, (Charles-Monica Press, Enugu, 2015) Appendix $\mathrm{H}$, Figure H2, p 301

[16] P. E. Chime, Chap 13 Total Orbital Bodies. In The Number And Distribution Of Planetary Orbits, Planets, Moons And Planetary Ring-Systems In The Solar System (Charles-Monica Press, Enugu, 2015) p $104-126$.

[17] P. E. Chime, The Number And Distribution Of Planetary Orbits, Planets, Moons And Planetary Ring-Systems In The Solar System, (Charles-Monica Press, Enugu, 2015) Appendix G, p 297 - 299.

[18] P. E. Chime, Chap 5 The Proposed Grading Of Planetary Orbits. In The Number And Distribution Of Planetary Orbits, Planets, Moons And Planetary Ring-Systems In The Solar System (Charles-Monica Press, Enugu, 2015) p 32 - 44.
[19] P. E. Chime, Chap 6 The Proposed Grading Of The Planets. In The Number And Distribution Of Planetary Orbits, Planets, Moons And Planetary Ring-Systems In The Solar System (Charles-Monica Press, Enugu, 2015) p 45 - 48.

[20] P. E. Chime, The Number And Distribution Of Planetary Orbits, Planets, Moons And Planetary Ring-Systems In The Solar System, (Charles-Monica Press, Enugu, 2015) Appendix I, Table I1, p 302 - 303.

[21] J. Warell, O Karlsson, A search for natural satellites of Mercury. Planetary and Space Science (2007), 55 (14): 20372041. Bibcode:2007P\&SS...55.2037W. doi: 10.1016/j.pss.2007.06.004.

[22] S. S. Sheppard, C. A. Trujillo, A survey for satellites of Venus. Icarus 202(2009) p $12-16$.

[23] M. H. Jones, D. Bewsher, D. S. Brown, Imaging of a Circumsolar Dust Ring Near the Orbit of Venus, Science 22 Nov 2013: Vol. 342, Issue 6161 p 960-963.

[24] S. S. Sheppard, D. Jewitt, J. Kleyna, A survey for outer satellites of Mars: Limits to completeness. The Astronomical Journal (Nov 2004) 128: p 2542 - 2546.

[25] M. R. Showalter, D. P. Hamilton, P. D. Nicholson. A deep search for Martian dust rings and inner moons using the Hubble Space Telescope. Planetary and Space Science (Aug 2006). Vol 54, Issues $9-10$, p $844-854$.

[26] P. E. Chime, Chap 28 The Fourteen Planets Of The Solar System. In The Number And Distribution Of Planetary Orbits, Planets, Moons And Planetary Ring-Systems In The Solar System (Charles-Monica Press, Enugu, 2015) p 252 - 260.

[27] P. E. Chime, Chap 20 Orbit Five. In The Planetary Orbits And Matter Of The Solar System: Analysis 1 (Charles-Monica Press, Enugu, 2012) p $220-224$.

[28] ] P. E. Chime, Chap 26 The Formation Of The Peripheral Bodies. In The Number And Distribution Of Planetary Orbits, Planets, Moons And Planetary Ring-Systems In The Solar System (Charles-Monica Press, Enugu, 2015) p 214 - 236.

[29] P. E. Chime, Chap 21 Orbit Eleven. In The Planetary Orbits And Matter Of The Solar System: Analysis 1 (Charles-Monica Press, Enugu, 2012

[30] P. E. Chime, Chap 20 The Number Of Planets In The Solar System At The Stage 3 Of Peripheral Bodies Formation. In The Number And Distribution Of Planetary Orbits, Planets, Moons And Planetary Ring-Systems In The Solar System (Charles-Monica Press, Enugu, 2015) p 166 - 176.

[31] P. E. Chime, Chap 22 The Number Of Moons In The Solar System At The Stage 3 Of Peripheral Bodies Formation. In The Number And Distribution Of Planetary Orbits, Planets, Moons And Planetary Ring-Systems In The Solar System (Charles-Monica Press, Enugu, 2015) p 182 - 189.

[32] P. E. Chime, Chap 10 Formation Of Peripheral Orbits And Peripheral Bodies In The Solar System. In The Number And Distribution Of Planetary Orbits, Planets, Moons And Planetary Ring-Systems In The Solar System (Charles-Monica Press, Enugu, 2015) p 56-62.

[33] P. E. Chime, Chap 12 The Distribution Pattern of Matter: The Planet's Masses. In The Planetary Orbits And Matter Of The Solar System: Analysis 1 (Charles-Monica Press, Enugu, 2012) p $155-166$. 
[34] P. E. Chime, Chap 24 The Formation Of Planets And Moons In The Solar System. In The Number And Distribution Of Planetary Orbits, Planets, Moons And Planetary RingSystems In The Solar System (Charles-Monica Press, Enugu, 2015) p $191-206$.

[35] P. E. Chime, Chap 25 The Formation Of The Planetary RingSystems. In The Number And Distribution Of Planetary Orbits, Planets, Moons And Planetary Ring-Systems In The Solar System (Charles-Monica Press, Enugu, 2015) p 208 213.
[36] IAU Definition of planet in the solar system. https://www.iau.org/news/pressreleases/detail/iau0603/

[37] Jean-Luc Margot, A Quantitative criterion for defining planets. Draft version October 11, 2015.

[38] K. D. Runyon, S. A. Stern, T. R. Lauer, W. Grundy, M. E. Summers, K. N. Singer, A geophysical planet definition. Lunar and planetary science XLVIII (2017). 